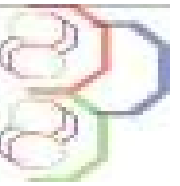

\title{
Amélioration de la résistance à l'usure des outils de travail des machines aratoires à disques
}

\author{
Viktor Aulin ${ }^{1}$, Warouma Arifa ${ }^{2}$, Borak Konstantin ${ }^{3}$ \\ 1Université Technique d'État de Kirovograd, Pravda Prospect, 70A -25006 Kirovograd, Ukraine, \\ 2Faculté d'Agronomie et des Sciences de l'Environnement, Université de Maradi (Niger). BP : 465 \\ 3Université Nationale Agroécologique de Zhytomyr, 7, Stary Blvd, Zhytomyr 10008, Ukraine \\ * Auteur de correspondance : warouma@yahoo.com
}

Original submitted in on 21st October 2014. Published online at www.m.elewa.org on 30th November 2014. http://dx.doi.org/10.4314/jab.v83i1.7

\section{RESUME}

Objectif : Ce travail a pour objectif de mettre en évidence la résistance à l'usure et l'effet d'auto affûtage des disques des machines aratoires fabriqués, consolidés et affutés différemment.

Méthodologie et résultats : Les essais au champ ont concerné cinq différents types de disques en série et trois différents types de disques expérimentaux montés sur un pulvériseur de marque UDA-4,5. II a été développé une méthode de mesure de l'usure linéaire et de la forme des disques lors des tests de performance après chaque 30 ha d'exploitation jusqu'à la durée limite d'exploitation. Les disques fabriqués en acier $65 \mathrm{G}$ et consolidés par décharge électrique avec affûtage simultané (angle d'affûtage de $30^{\circ}$ ) ont présenté les meilleurs résultats : une durée d'exploitation de 178 ha, une résistance à l'usure 1,76 fois supérieure à celle des disques en série et l'effet de l'auto-affûtage s'est observé tout au long de l'exploitation.

Conclusion et application des résultats : Les disques fabriqués en acier $65 \mathrm{G}$ et consolidés par décharge électrique avec affûtage simultané (angle d'affûtage de $30^{\circ}$ ) résistent plus à l'usure et travaillent sans changement de forme. Le temps d'exploitation a duré jusqu'au franchissement de l'état limite sans que les disques soient affûtés. Les résultats obtenus peuvent être appliqués lors de la fabrication des disques, ainsi que lors de la rectification des disques en cours de l'exploitation.

Mots-clés : usure, consolidation par décharge électrique, disques, sol, résistance à l'usure, auto-affûtage.

\section{Improvement of resistance to wearing down of hard discs of plowing machines}

\section{ABSTRACT}

Objective: This work aimed to highlight the wear resistance and self sharpening effect of disc plowing machinery manufactured, consolidated and sharpened differently.

Methodology and Results: The field trials have involved five different types of discs in series and three other different types of experimental disks mounted on a pulverizer of UDA-4, 5 brand. It has been developed a method for measuring the linear wear and the shape of the discs during performance testing after each operating of 30 ha the operating time limit. Discs made from $65 \mathrm{G}$ steel and consolidated by electric discharge with simultaneous sharpening (sharpening angle of $30^{\circ}$ ) has shown the best results which are: an operating duration of178ha, a wear resistance 1.76 times greater than that of discs in series and the effect of selfsharpening has been observed throughout the exploitation. 
Conclusion and application results: The discs made from $65 \mathrm{G}$ steel and consolidated by with simultaneous sharpening (sharpening angle of $30^{\circ}$ ) are more resistant to the wear and work without changing the shape. The operating time lasted until crossing the limit state without sharpening of discs. These results can be applied during the production of discs, and when repairing discs during operation.

Keywords: wear, consolidation by electric discharge, discs, soil, wear resistance, self-sharpening.

\section{INTRODUCTION}

La principale raison de la perte de la capacité de travail des pièces des machines agricoles est l'usure. Les défaillances dues à l'usure dans les conditions modernes représentent 80 à $90 \%$ du total des défaillances (Warouma, 1999). L'amélioration de la résistance à l'usure des pièces fonctionnant dans les conditions d'usure abrasive est l'une des principales tâches de l'ingénierie moderne (Tkachev, 1995; Khrouchtchev et al., 1970). Les machines aratoires à disques (MAD) constituent environ 40\% du total des machines agricoles (Aulin et al., 2008; Tikhonov et al., 2010; Strelbitsky, 1978). Les disques sphériques crénelés sont plus largement utilisés, car ils réagissent intensivement dans le sol et coupent mieux les résidus de cultures (Borak, 2013). Lors du travail des disques sphériques crénélés, il ne se produit aucune accumulation des racines et des tiges à l'avant des disques. Suite à l'usure, les dimensions nominales du disque et sa forme subissent des modifications qui affectent de manière significative la qualité de la réalisation du processus technologique (Aulin et al., 2013; Sidorov, 2007 ; Bernstein., 2002 ). Par conséquent, il est nécessaire de conserver la forme et d'améliorer la résistance à l'usure des outils de travail (OT) des machines aratoires à disques (MAD). Pour la fabrication des pièces travaillant dans les conditions d'usure abrasive, selon (Bernstein, 2002 ; Sineokov et al., 1977, Kostecki et al., 1976 ; Nartov., 1972), il est recommandé d'utiliser les matériaux suivants: acier à base de manganèse (30G, 50G, 65G, 110G6X3L), acier allié au chrome (38CrA, 40Cr, $45 \mathrm{Cr}, \mathrm{Cr} 12$, Ni12F1, Ni6BF), acier allié multicomposant et alliages comme 12CrNi3A, 17CrG2CFP, 08C18Ni10Ti (Rabinovich, 1962; Tkachev, 1995, Mikhalchenkov et al., 2006) et des alliages frittés solides tels que BK6, BK8, BK15, BK20 (Severnev, 1972 ; Sidorov, 2007). L'analyse des OT des MAD a montré une faible efficacité en raison de l'usure rapide du diamètre extérieur, de l'émoussement du tranchant, ainsi que du changement de la surface des dents (disques à crénelures), ce qui conduit à l'augmentation de la consommation en carburant et en lubrifiant, à la dégradation de la qualité du travail du sol, à la nécessité d'effectuer un affûtage supplémentaire des outils de travail (OT) et au remplacement de pièces usées ou à leur restauration (Mikhalchenkov et al., 2006 ; Rabinovich, 1962, Sineokov et al., 1977).

Le choix de la méthode d'augmentation de la résistance à l'usure doit tenir compte du fait que l'usure au niveau des OT des MAD a un caractère sélectif (local) en raison de la nature irrégulière de la pression du sol sur les OT (Sidorov, 2007 ; Viktor Aulin et al., 2013). Lors du choix de la méthode de consolidation des disques des machines aratoires (MA), il est important de tenir compte de la nécessité de la réalisation de l'effet d'auto-affûtage. Des études ont montré que, l'effet d'auto-affûtage des disques se produit lorsqu'on effectue la consolidation sur la face intérieure du disque et l'aiguisage de la lame du coté extérieur (Mikhalchenkov et al., 2006) . Par contre, la consolidation du disque du coté extérieur et l'aiguisage du coté interieur donnent aussi un bon résultat. Aussi l'auto-affûtage est possible, mais il faut que le rapport de la dureté de la couche résistante à l'usure et du métal de base dépasse 7,0 (Khrouchtchev et al., 1970). Ainsi, pour la problématique concernant la consolidation des OT des MAD avec la mise en œuvre de l'effet d'autoaffûtage, les chercheurs n'ont pas de point de vue commun. C'est pourquoi d'autres études qui tiennent compte du type de sol, de son état, et de ses propriétés physiques sont nécessaires. Les possibilités constructives pour améliorer la résistance à l'usure des pièces des machines sont très variées, mais se résument principalement à améliorer le régime de fonctionnement des pièces (Dudak, 2007 ; Aulin, 2013). Afin d'améliorer la résistance à l'usure des OT, l'angle d'affûtage des 


\section{Aulin et al. J. Appl. Biosci. 2014 Amélioration de la résistance à l'usure des outils de travail des machines aratoires à disques}

disques consolidés doit être de l'ordre de $28^{\circ}$ à $30^{\circ}$ (Bernstein, 2002). Cette étude a pour principal objectif l'identification des matériaux et des

\section{MATERIEL ET METHODE}

Matériel : II est composé de: un dispositif pour la consolidation par décharge électrique (CDE) de marque 01.10.016A, un pulvériseur universel de marque UDA4,5 , un dispositif pour la mesure de l'usure linéaire des disques et des disques crénelés de marque UD 7.5.00 fabriqués en acier $65 \mathrm{G}, 45, \mathrm{X} 12$ et $28 \mathrm{MnB} 5$ et consolidés par différentes méthodes.

Méthode : La consolidation des disques a été effectuée sur le dispositif de la CDE (fig.1) au laboratoire de la Faculté d'exploitation et de réparation des machines de l'Université d'État Technique de Kirovograd (Ukraine). technologies de fabrication et de consolidation qui résistent mieux à l'usure.

Les expérimentations des OT se sont déroulées entre 2013 et 2014 dans un champ d'essais, en zone de steppe de l'Université Nationale Agroécologique de Jitomir (Ukraine). L'humidité du sol est de 9 à $25 \%$ dans les périodes de travail sur le terrain pendant l'été, le printemps et l'automne. La vitesse de travail du pulvériseur était de 8 à $15 \mathrm{~km} / \mathrm{h}$ avec une profondeur de labour comprise entre 8 et $20 \mathrm{~cm}$. Lors de l'expérimentation, il a été procédé aussi à la surveillance de l'effet d'auto-affûtage.

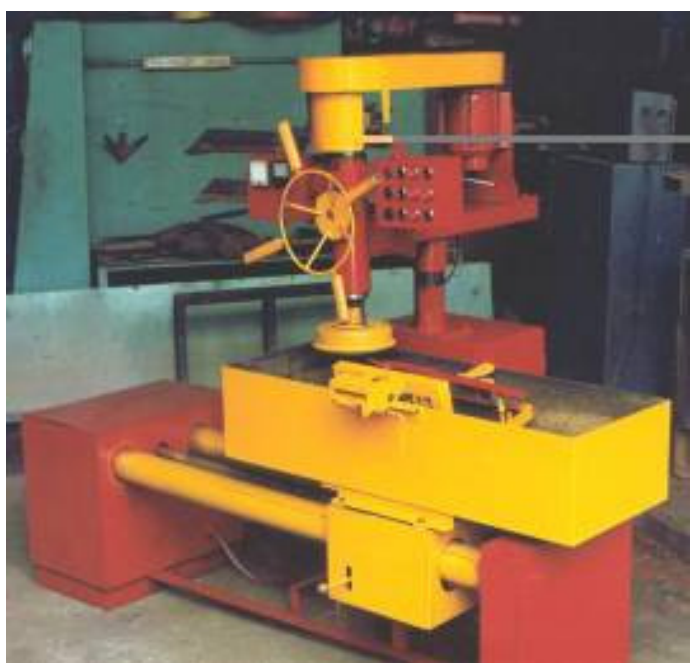

a)

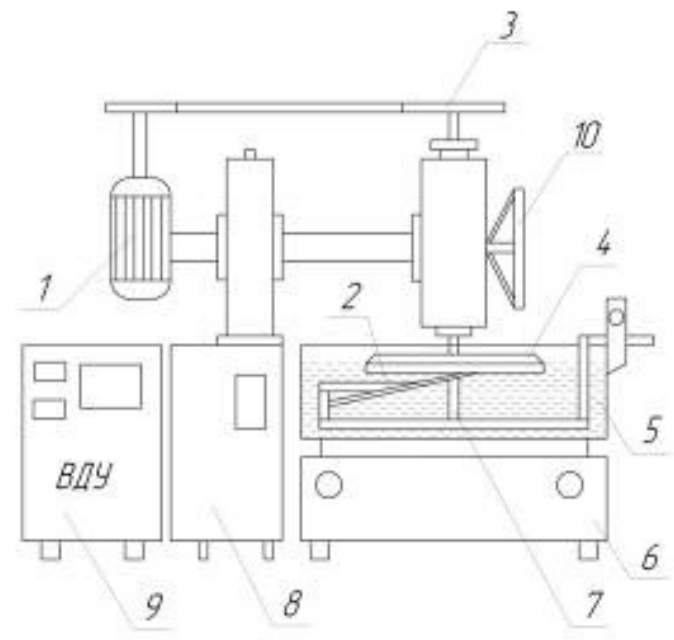

b)

Figure 1: Vue générale (a) et schéma (b) du dispositif de la consolidation par décharge électrique de marque 01.10.016A: 1 - moteur électrique; 2 - pièce; 3 - entraînement par courroie; 4 - électrode-outil; 5 - salle de bain; 6 - entraînement du bain ; 7 - berceau; 8 - armoire de commande; 9 - source d'alimentation, 10 - réglage du volant. La couche consolidée est obtenue par la fonte du métal de la pièce au point de contact et s'amène dans le bain. La surface préchauffée de la pièce au moyen d'un milieu diélectrique se refroidit rapidement, subissant ainsi une trempe jusqu'à une dureté HRC de 40 à 65 . La consolidation s'est effectuée sur la surface externe des disques.

Tous les disques ont été installés en groupes de deux trains (avant et arrière) du pulvériseur universel UD7,5.00. Lors de l'expérimentation, les disques de mêmes types ont été connectés sur un même train de disques, en même temps les autres trains du pulvériseur ont été équipés avec des disques de types différents. Cela est acceptable dans la mesure où chaque train de disque a un système de charge indépendant. L'usure linéaire du diamètre du disque a été déterminée par le dispositif représenté schématiquement sur la figure 2. 


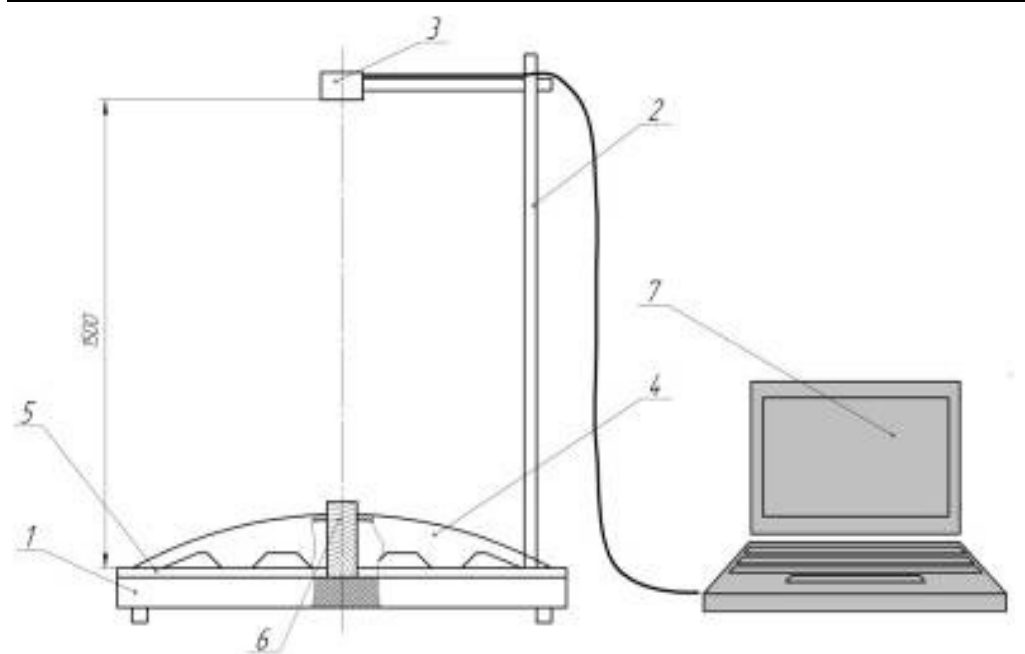

a)

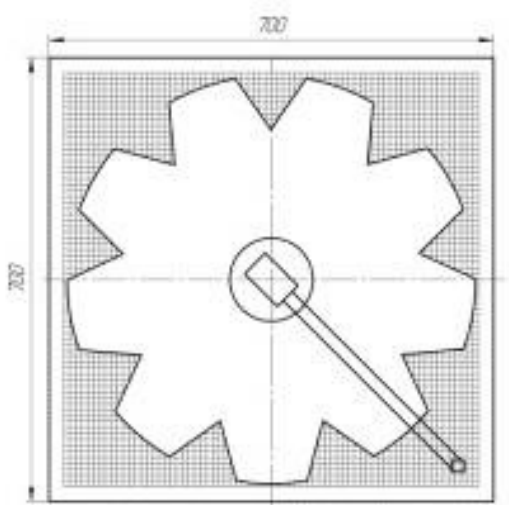

b)

Figure 2 : Dispositif pour la détermination de l'usure linéaire du diamètre des disques (a) et vue de dessus (b): 1 - base, 2 - trépied, 3 - appareil photo numérique, 4 - disque, 5 - grille à coordonnées, 6-ordinateur portable.

Les mesures ont été effectuées dans l'ordre suivant: - Le disque est installé sur l'appareil et photographié, les données sont transmises à un ordinateur et traitées à l'aide du logiciel 3D V9;

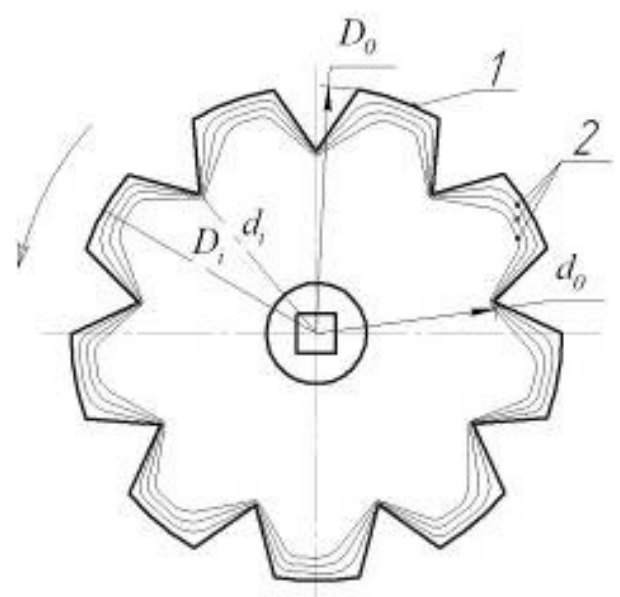

- La différence de la distance entre les contours initiaux et finaux du disque est déterminée à l'aide d'un ordinateur (fig. 3).

Figure 3: Schéma de détermination de l'usure des outils de travail des machines aratoires à disques: Do, do respectivement les diamètres initiaux intérieur et extérieur, $\mathrm{Di}$, di -, respectivement, les diamètres externe et interne après un certain temps de fonctionnement.

Cette méthode permet d'observer la dynamique du changement d'usure linéaire et de la forme des OT des MAD lors des essais d'exploitation. Pour l'expérimentation, il a été utilisé des disques de marque UD 7.5.00 (fig.4) et dont les caractéristiques techniques sont indiquées au tableau 1. 


\section{Aulin et al. J. Appl. Biosci. 2014 Amélioration de la résistance à l'usure des outils de travail des machines aratoires à disques}

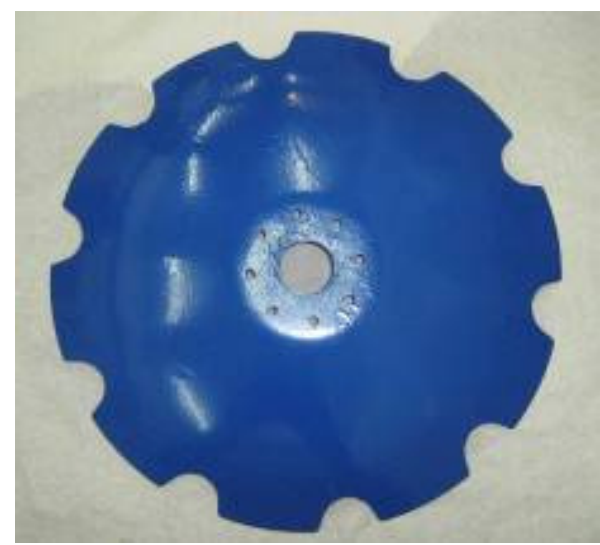

Figure 4 : Vue générale du disque UD 7.5.00

Tableau 1 : Fiche technique du disque UD 7.5.00

\begin{tabular}{|c|c|c|c|c|c|}
\hline Type de disque & $\begin{array}{c}\text { angle d'affûtage, } \\
\text { degrés }\end{array}$ & Poids, $\mathrm{kg}$ & Diamètre, $\mathrm{mm}$ & $\begin{array}{l}\text { Nombre de } \\
\text { dents }\end{array}$ & $\begin{array}{c}\text { Largeur des } \\
\text { dents, } \mathrm{mm}\end{array}$ \\
\hline UD 7.5.00 & 17 & $14,400 \pm 0,1$ & 655 & 10 & 130 \\
\hline
\end{tabular}

\section{RESULTATS}

La vue générale du disque en acier $65 \mathrm{G}$ renforcé par décharge électrique est montrée sur la Figure 5.

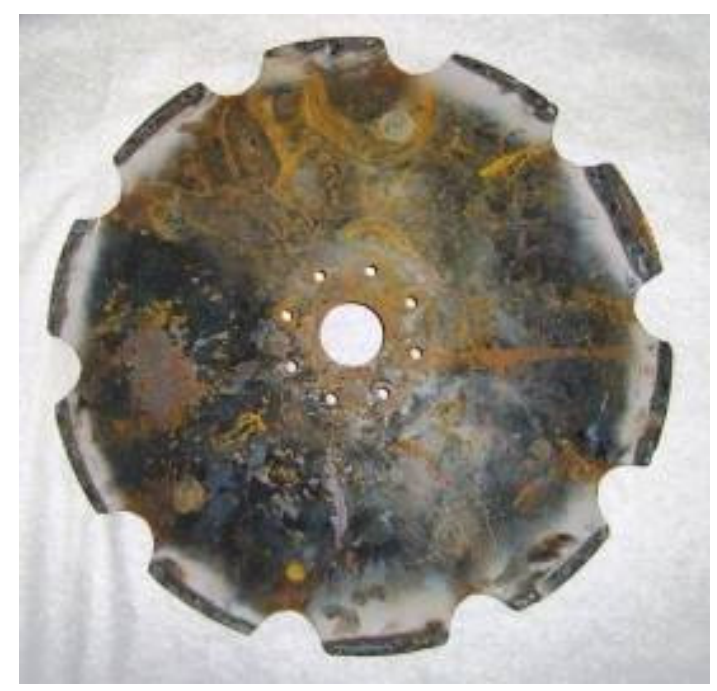

Figure 5: Vue générale du disque UD 7.5.00 en acier 65G renforcé par décharge électrique, avec un affûtage simultané (angle d'affûtage $30^{\circ}$ )

La dynamique de l'usure linéaire des disques des MA en série et expérimentaux est représentée sur la figure 6. 


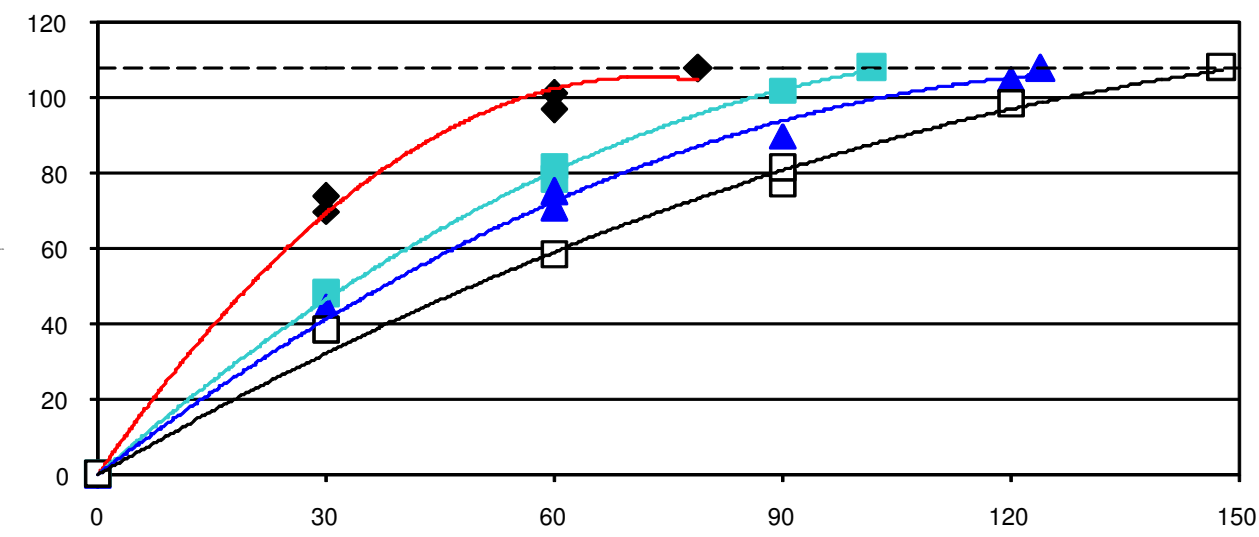

Durée d'exploitation pour un disque, ha

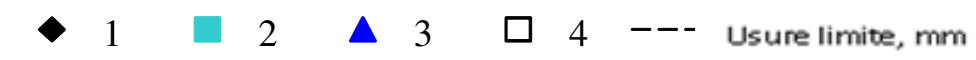

a)

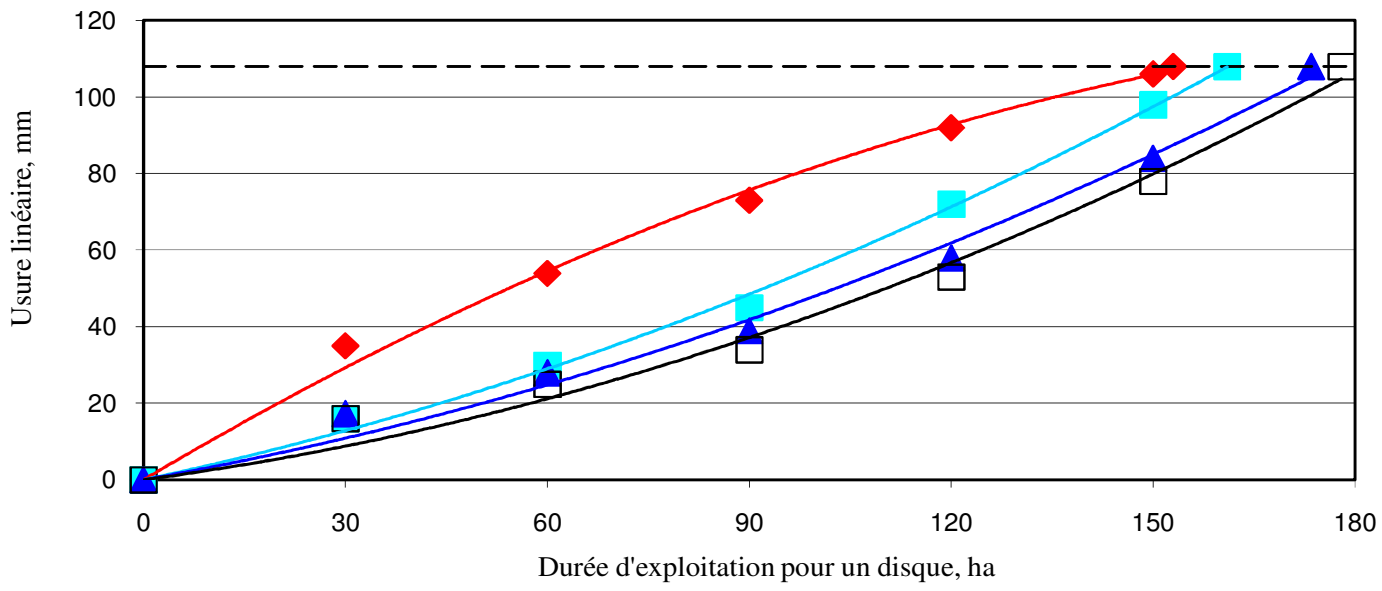

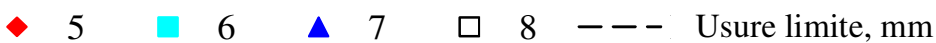

b)

Figure 6 : Dépendance de l'usure des outils de travail des machines aratoires à disques en fonction de la durée d'exploitation. (a) disques non auto-affûtants; b) disques auto-affûtants : 1 - disques en série fabriqués en acier 45; 2 disques en série fabriqués en acier 65G; 3 - disques en série fabriqués en acier $65 \mathrm{G}$ avec une trempe volumétrique à une température de 810 à $830^{\circ} \mathrm{C}$ et un revenu moyen soumis à une température de 460 à $480^{\circ} \mathrm{C} ; 4$ - disques en série fabriqués en acier $\mathrm{X} 12 ; 5$ - disques en série fabriqués en acier $28 \mathrm{MnB} 5 ; 6$ - disques expérimentaux fabriqués en acier $65 \mathrm{G}$ et renforcés par décharge électrique avec affûtage simultané (angle d'affûtage $17^{\circ}$ ); 7 - disques expérimentaux fabriqués en acier $65 \mathrm{G}$ et consolidés avec l'électrode T-590; 8 - disques expérimentaux fabriqués en acier $65 \mathrm{G}$ et consolidés par décharge électrique avec affûtage simultané (angle d'affûtage de $30^{\circ}$ ). 
La figure 6 montre que l'état limite d'usure des disques testés se constate avec la diminution du diamètre jusqu'à $546 \mathrm{~mm}$ pour un diamètre initial de $655 \mathrm{~mm}$. Ainsi, les disques en série fabriqués en acier 45 ont la plus petite durée d'exploitation évaluée à 79 ha. Par contre, les disques expérimentaux fabriqués en acier $65 \mathrm{G}$ et consolidés par décharge électrique avec affûtage simultané (angle d'affûtage de $30^{\circ}$ ) ont présenté la plus grande durée d'exploitation évaluée à 178 ha. Pour déterminer la nécessité d'affûtage des disques, les valeurs de référence des critères de l'état limite des lames, en vertu desquelles la nécessité d'affûtage s'impose, sont définies au tableau 2.

Tableau 2: Valeur approximative des critères de l'état limite de la lame du disque du pulvériseur

\begin{tabular}{|l|c|}
\hline Désignation de l'indicateur & Valeur \\
\hline Angle d'affutage de la lame, degrés & 36 à 42 \\
\hline Épaisseur de la partie tranchante prise à la distance de $1 \mathrm{~mm}$ du bout de la lame, $\mathrm{mm}$ & 1,5 à 2,2 \\
\hline
\end{tabular}

Le diagramme de la durée d'exploitation jusqu'à l'état limite des disques fabriqués avec différents types de matériaux et différentes méthodes de durcissement, est représenté à la figure 7 .

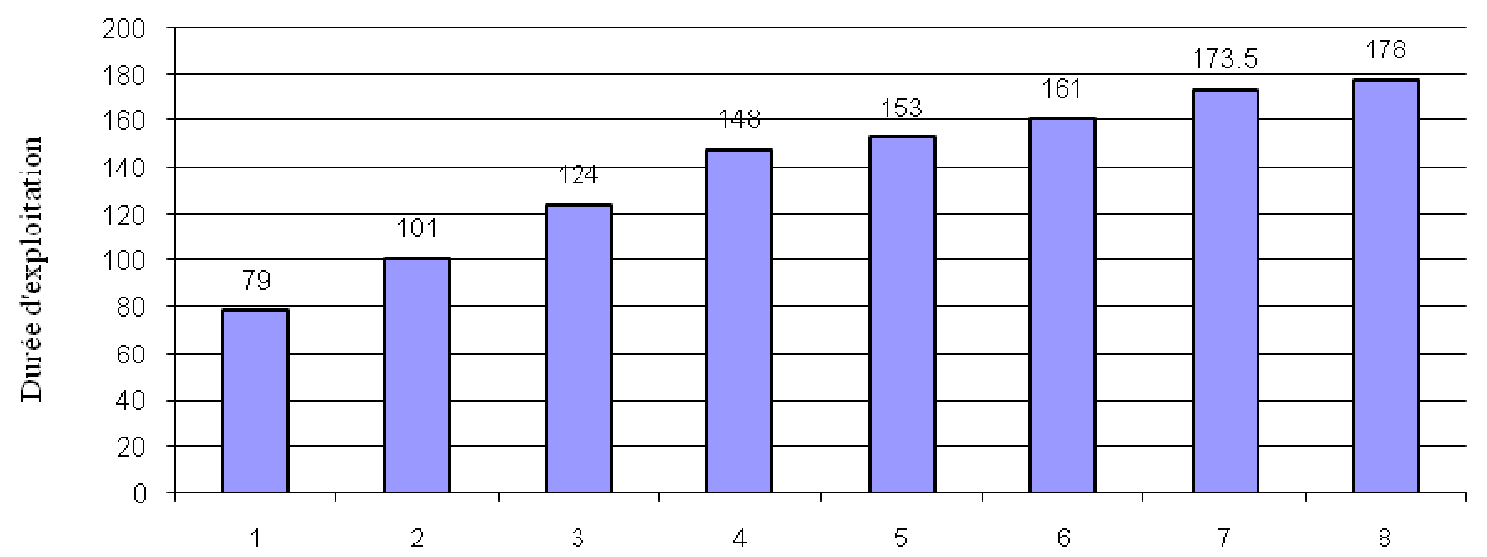

Figure 7 : Durée d'exploitation de différents types de disques . 1-disques en série fabriqués en acier 45; 2 - disques en série fabriqués en acier $65 \mathrm{G} ; 3$ - disques en série fabriqués en acier $65 \mathrm{G}$ avec une trempe volumétrique à une température de 810 à $830^{\circ} \mathrm{C}$ et un revenu moyen soumis à une température de 460 à $480^{\circ} \mathrm{C} ; 4$ - disques en série fabriqués en acier X12;5-disques en série fabriqués en acier $28 \mathrm{MnB} 5 ; 6$ - disques expérimentaux fabriqués en acier $65 \mathrm{G}$ et renforcés par décharge électrique avec affûtage simultané (angle d'affûtage 17 ${ }^{\circ}$; 7 - disques expérimentaux fabriqués en acier $65 \mathrm{G}$ et consolidés avec l'électrode T-590; 8-disques expérimentaux fabriqués en acier $65 \mathrm{G}$ et consolidés par décharge électrique avec affûtage simultané (angle d'affûtage de $30^{\circ}$ ).

Comme le montre la figure 7 , les disques fabriqués en acier $65 \mathrm{G}$ et consolidés par décharge électrique avec affûtage simultané (angle d'affûtage de $30^{\circ}$ ) ont une durée d'exploitation de 178 ha. Les disques en acier 45 ont été affûtés durant leur exploitation après 30 et 60 ha de labour. La même opération a été effectuée pour les disques en acier $65 \mathrm{G}$ et pour ceux en acier $65 \mathrm{G}$ avec trempe volumétrique à une température de 810 à $830^{\circ} \mathrm{C}$, un revenu moyen et exposé à une température de 460 à $480^{\circ} \mathrm{C}$ après un labour de 60 ha. Les disques fabriqués en acier $28 \mathrm{MnV} 5$ lors de fonctionnement commencent à être émoussés après une exploitation de 120 ha par disque. Chez les disques renforcés par décharge électrique avec un angle d'aiguisage de $17^{\circ}$, il a été observé un excès de sortie de la couche dure et sa cassure lors de l'interaction avec des éléments durs dans le sol. Les disques consolidés avec l'électrode T -590 ont commencé à s'émousser après un labour de 90 ha par disque. La méthode la plus optimale est la consolidation par décharge électrique avec un angle d'affutage de $30^{\circ}$. Avec un tel angle, pendant l'exploitation, il a été observé l'effet d'auto-affûtage de la lame des disques (fig.8), ce qui a permis d'augmenter le temps de fonctionnement des disques consolidés par décharge électrique jusqu'à 


\section{Aulin et al. J. Appl. Biosci. 2014 Amélioration de la résistance à l'usure des outils de travail des machines aratoires à disques}

l'annonce de l'état limite d'usure. La résistance à l'usure des disques consolidés par décharge électrique avec aflûtage simultané (angle d'aflûtage de $30^{\circ}$ ) est de 1,76 fois supérieure à celle des disques en série fabriqués avec le même matériel.

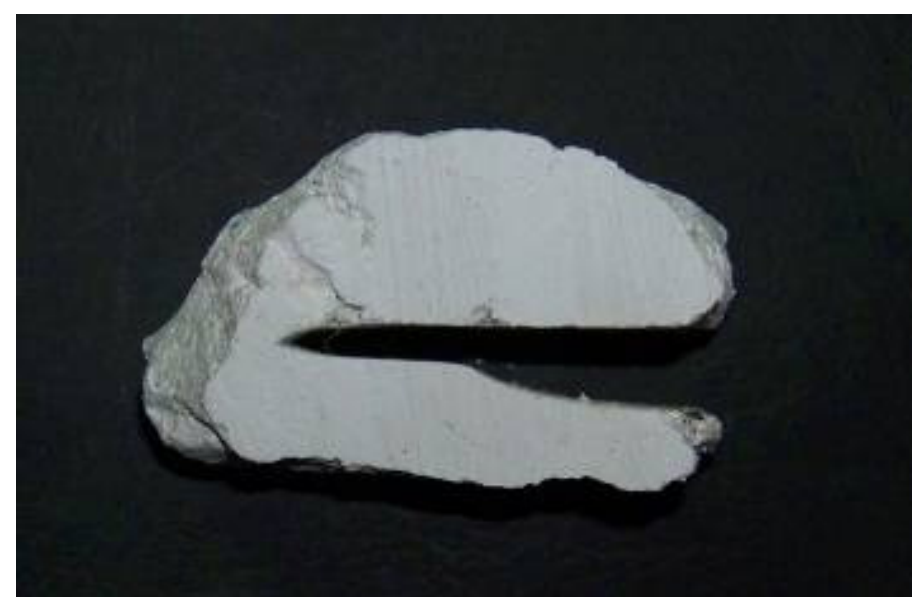

Figure 8 : Vue générale du profil de la lame du disque consolidé par décharge électrique (après 60 ha d'exploitation).

\section{DISCUSSIONS}

En analysant les graphiques de la figure 6, nous pouvons conclure que la dépendance de l'usure en fonction de la durée de fonctionnement pour les disques des MA (fig.6,a) non consolidés est caractérisée par une monotonie et une décroissance continue jusqu'à l'état limite d'usure. Chez les disques des MA consolidés (Fig. $6, b)$, la non homogénéité du matériau en profondeur conduit au changement de la nature de l'usure. Chez tous les disques testés, l'état limite d'usure se constate avec la diminution du diamètre jusqu'à $546 \mathrm{~mm}$. Pour les disques en série fabriqués en acier $45,65 \mathrm{G}$ et disques fabriqués en acier $65 \mathrm{G}$ trempé, le critère du premier cas de l'état limite est l'augmentation de l'angle d'affûtage et du rayon de courbure, la création d'une large face occipitale qui à leur tour ont conduit au non respect des exigences agrotechniques, et le critère du deuxième cas de l'état limite est l'usure diamétrale. Au niveau de tous les autres disques, l'usure diamétrale est le critère du premier cas de l'état limite. Comme le montre la figure 6 , les disques fabriqués en acier $65 \mathrm{G}$ et renforcés par décharge

\section{CONCLUSION.}

Les études théoriques ont permis d'asseoir les conditions d'amélioration de la résistance à l'usure et d'auto-affûtage des disques crénelés des MA. Les résultats des essais ont montré que la résistance à l'usure des disques fabriqués en acier $65 \mathrm{G}$ et consolidés par décharge électrique avec affûtage simultané (angle d'affûtage de $30^{\circ}$ ) a augmenté de 1,76 fois sans changement de forme. Aussi, la durée d'exploitation a augmenté jusqu'au électrique avec un affûtage simultané (angle d'affûtage de $30^{\circ}$ ) sont plus endurants. Cette endurance est 1,76 fois supérieure à celle des disques en série fabriqués avec le même type de matériel de base. Ces résultats sont différents avec ceux de Mikhalchenkov et al., 2006 et de Tikhonov et al., 2010 où la résistance à l'usure des disques expérimentaux est respectivement de 2,2 à 2,8 fois inférieure à celle, qui est en série, et 2,0 à 3,5 fois supérieure à la résistance à l'usure des socs en série. Ces différences significatives s'expliquent dans un premier temps du fait de différentes méthodes de consolidation des outils de travail et d'autre part de leur exploitation dans différentes conditions de sol. Les essais expérimentaux ont montré que l'angle d'affûtage pour la méthode la plus optimale (consolidation par décharge électrique) est de $30^{\circ}$. Ces chiffres sont semblables avec ceux de Bernstein, 2002 où l'angle optimal est compris entre 28 et $30^{\circ}$, mais diffère avec ceux obtenus par Dudak, 2007 où l'angle optimal d'affûtage est compris entre 18 et $25^{\circ}$.

franchissement de l'état limite. Le phénomène d'autoaffûtage a été observé tout au long de la durée d'exploitation de ces disques et la forme du disque est restée presque inchangée. Cette méthode de consolidation peut être utilisée lors de la fabrication et / ou de la rectification des disques du pulvériseur. Cependant, il serait intéressant d'effectuer les mêmes essais avec les disques sans crénelures car ils ont des 
réactions moins intensives sur le sol que les disques à RÉFÉRENCES BIBLIOGRAPHIQUES

Aulin V.V., 2013. État de l'auto-organisation du sol et de la régularité de l'usure des organes de travail des machines aratoires / problèmes de tribologie.- Khmelnitsky.- KNU 1: 114 -119.

Aulin V.V. et Borak K.V., 2013. Amélioration de la résistance à l'usure des organes de travail à disques des machines aratoires avec la mise en œuvre de l'effet d'auto-affûtage / Problèmes de synergie dans la tribologie, tribo-electrochimie, conduite des matériaux et la mécatronique. Actes de la XII Conférence scientifique et pratique internationale. Novocherkask. YURGPU (NPI) : 50 - 58.

Aulin V.V., Zaitsev O.Y., Julay O.Y., Bobritsky V.M., Lyssenko S.V.; zayavnik Aulin V.V., 2008. Organes de travail à disques / Licence N 33420 Ukraine, MPK A01V7/00.-U 2008 00971.- dépôt demande : 28.01.2008.- publication : 25.06.2008, bulletin $\mathrm{N}^{\circ} 12$.

Bernstein D.B., 2002. Usure par abrasion de la lame des disques et la performance du pulvériseur// Tracteurs et machines agricoles. - M: génie, -6 : 39-42.

Borak K.V., 2013. Amélioration de la résistance à l'usure des disques de travail du sol par décharge électrique: Travaux de thèse de doctorat. 217pp.

Dudak S.M., 2007. Outils de travail à disques: Principaux paramètres et caractéristiques // Mécanisation et électrification de l'agriculture $91:$ 368-371.

Khrouchtchev M.M. et Babichev M.A., 1970. Usure abrasive / MA.- M.: Science, - 252 pp.

Kostecki B.I., Strel'nikov B.P.,Tatsiy V.G., 1976. Problèmes de la friction et de l'usure. - K: Technique $10: 10-15$.

Mikhalchenkov A.M., Tyureva A.A., Parshikov P.A., 2006. Amélioration de la résistance à l'usure des disques par la consolidation des zones les plus probables d'usure // Construction, exploitation et fiabilité des machines agricoles/ Recueil des travaux scientifiques. - Briansk: Académie Agricole Nationale de Briansk- 1: 234-239.

Nartov P.S., 1972. Organes de travail à disques.Voronezh.- $184 \mathrm{pp}$.

Rabinovich A.S, 1962. Socs auto-affûtant et autres outils de travail du sol.- M:. GOSNITI. 106 pp.

Severnev M.M., 1972. Usure des pièces des machines agricoles. - L.. Kolos. 288 pp.

Sidorov S.A., 2007. Amélioration de la longévité et de la performance des organes de travail des crénelures.

machines et outils aratoires utilisés dans l'agriculture et la sylviculture: Travaux de thèse de doctorat. - M. 441 pp.

Sineokov G.P. et Panov I.M., 1977. Théorie et calcul de machines aratoires. - M: Construction des machines agricoles. $328 \mathrm{pp}$.

Strelbitsky V.F., 1978. Machines de travail du sol à disques. - M:. Génie mécanique. 135 pp.

Tikhonov D.A. et Sidashenko A.l., 2010. Système de technologie de réparation de la partie coupante des organes de travail à disques des machines aratoires/ Amélioration de la performance des machines et outils: Travaux des doctorants et Enseignants à la 4ème Conférence Nationale Scientifique et Pratique de l'Ukraine. Kirovograd: KNTU : 180-183.

Tkachev V.N., 1995. Performances des outils dans les conditions d'usure abrasive. - M:. Génie mécanique. $336 \mathrm{pp}$.

Viktor A. et Warouma A., 2013. Étude de l'état de contrainte du sol et des organes de travail des machines aratoires et leurs effets sur la résistance à la traction. Journal of Applied Biosciences. 72:5883- 5891.

Warouma A., 1999. Rectification et consolidation des pièces des machines agricoles à l'aide $d u$ rechargement en poudre au chalumeau à gaz des revêtements céramico-métalliques.Travaux de thèse.-Université Technique d'État de Kirovograd. 137pp. 